\begin{tabular}{|c|c|}
\hline Title & $\begin{array}{l}\text { Temperature dependence of inter-dot electron-spin transfer among laterally coupled excited states in high-density } \\
\text { InGaA s quantum dots }\end{array}$ \\
\hline Author(s) & Sato, Shino; Hiura, Satoshi; Takay ama, Junichi; Muray ama, A kihiro \\
\hline Citation & $\begin{array}{l}\text { Journal of A pplied Physics, } 127(4), 043904 \\
\text { https://doi.org/10.1063/1.5134002 }\end{array}$ \\
\hline Issue Date & 2020-01-31 \\
\hline Doc URL & http:/hdl.handle.net/2115/80323 \\
\hline Rights & $\begin{array}{l}\text { This article may be downloaded for personal use only. A ny other use requires prior permission of the author and AIP } \\
\text { Publishing. This article appeared in J. A ppl. Phys. } 127(4), 043904 \text { (2020) and may be found at } \\
\text { http://doi.org/10.1063/1.5134002. }\end{array}$ \\
\hline Type & article \\
\hline File Information & 1.5134002.pdf \\
\hline
\end{tabular}

Instructions for use 


\section{Temperature dependence of inter-dot electron-spin transfer among laterally coupled excited states in high-density InGaAs quantum dots}

Cite as: J. Appl. Phys. 127, 043904 (2020); https://doi.org/10.1063/1.5134002

Submitted: 29 October 2019 . Accepted: 09 January 2020 . Published Online: 23 January 2020

Shino Sato, Satoshi Hiura (D), Junichi Takayama (D), and Akihiro Murayama
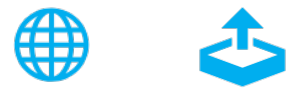

\section{ARTICLES YOU MAY BE INTERESTED IN}

Improving the performance of light-emitting diodes via plasmonic-based strategies Journal of Applied Physics 127, 040901 (2020); https://doi.org/10.1063/1.5129365

Magnetic reversal in rare-earth free $\mathrm{Mn}_{4-}{ }_{-} \mathrm{Ni}_{x} \mathrm{~N}$ epitaxial films below and above $\mathrm{Ni}$ composition needed for magnetic compensation around room temperature Journal of Applied Physics 127, 043903 (2020); https://doi.org/10.1063/1.5128635

Nanoscale electro-thermal interactions in AIGaN/GaN high electron mobility transistors Journal of Applied Physics 127, 044502 (2020); https://doi.org/10.1063/1.5123726

\section{Lock-in Amplifiers up to 600 $\mathrm{MHz}$}
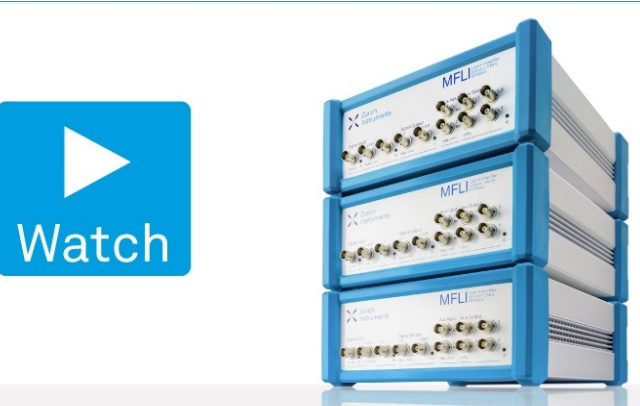


\title{
Temperature dependence of inter-dot electron-spin transfer among laterally coupled excited states in high-density InGaAs quantum dots
}

Cite as: J. Appl. Phys. 127, 043904 (2020); doi: 10.1063/1.5134002

Submitted: 29 October 2019 - Accepted: 9 January 2020 .

Published Online: 23 January 2020

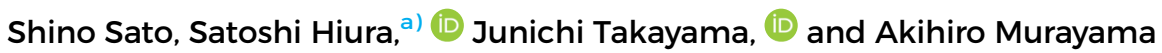

\author{
AFFILIATIONS \\ Faculty of Information Science and Technology, Hokkaido University, Kita 14, Nishi 9, Kita-ku, Sapporo 060-0814, Japan
}

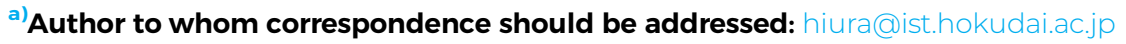

\begin{abstract}
The temperature dependence of interdot spin-transfer dynamics at laterally coupled excited states (ESs) in high-density InGaAs quantum dots (QDs) was studied using spin- and time-resolved photoluminescence spectroscopy. At low temperatures below $100 \mathrm{~K}$, temporary suppression of electron-spin polarization decay due to selective relaxation of minority spins from emissive ESs to lower-energy states in neighboring QDs was observed. In the temperature range from $20 \mathrm{~K}$ to $140 \mathrm{~K}$, thermal activation of electron spins from lower-energy QD states to higher-energy states via interdot transfer prevents the aforementioned selective relaxation of minority spins, leading to a faster decay of electron-spin polarization during light emission. At high temperatures above $140 \mathrm{~K}$, reinjection of depolarized electron spins from barriers after thermal escape from QD ESs accelerates the further decay of the electron-spin polarization, wherein the electron spins can be activated via ladderlike interdot transfer. These findings indicate that the suppression of reinjection of electron spins from barriers in a high-density QD system is crucial for maintaining high electron-spin polarization during light emission at high temperatures.
\end{abstract}

Published under license by AIP Publishing. https://doi.org/10.1063/1.5134002

\section{INTRODUCTION}

Semiconductor quantum dots (QDs) have been widely investigated as an optically active layer in ultralow power consumption light-emitting devices, owing to their energetically discrete density of the states of the carriers. In addition, III-V semiconductor QDs such as $\operatorname{In}(\mathrm{Ga})$ As have attracted much attention for application in optical spin devices that use electron-spin states and the resultant circular polarization. The spin relaxation in these QDs can be significantly suppressed, ${ }^{1,2}$ leading to a spin lifetime that is long compared to the radiative decay time. ${ }^{3,4}$ This means that it is possible to create and transfer the spin polarization via light emission. For the development of QD-based optical devices, high-density QDs are essential for strong light emission and high optical gain. Moreover, for the addition of spin functions, an increase in the QD density can suppress the spin-state filling effect due to Pauli blocking at the QD emissive states, ${ }^{7}$ whereby this spin-state filling can reduce the spin polarization during light emission. Recently, electron-spin polarization decay at QD excited states (ESs) was found to be temporarily suppressed in laterally coupled highdensity InGaAs QD ensembles. ${ }^{8,9}$ Size-modulated InGaAs QD nanocolumns with electron wavefunction coupling among the QDs along the stacking direction demonstrated amplification of electron-spin polarization in excess of $80 \%$ for coupled ESs. ${ }^{10}$ The minority spins can relax when the appropriate excitation density condition is achieved, whereas the majority ones cannot relax owing to Pauli blocking. Consequently, ES spin polarization can be enhanced. The suppression of spin polarization decay and further amplifications at QD ESs were observed at $6 \mathrm{~K}$. At this low temperature, electron spins can relax from higher-energy small QDs to lower-energy large ones via wavefunction coupling (tunneling) among neighboring QDs. ${ }^{11,12}$ However, at higher temperatures, thermal excitation of spin-polarized carriers can occur. In the case of coupled QDs, the interdot thermal spin transfer from lowerenergy states to higher-energy states can potentially affect the spinpolarized emissions. This temperature-dependent spin-transfer dynamics and the resultant spin-polarized emission properties in coupled QD ensembles have not been adequately investigated to date. A quantitative understanding of the effect of thermal spin excitation on spin dynamics around QDs, such as thermal excitation into barriers and spin transfer among QDs, is important for 
the development of optical spin devices based on high-density QDs. This thermal spin behavior will assist in the determination of an appropriate design for the quantum structures that surround each QD in the high-density QD system, with respect to the roomtemperature operation of spin-functional QD optical devices.

In this study, the temperature dependence of the spin-transfer dynamics at ESs of laterally coupled InGaAs QDs was investigated. At low temperatures, temporary suppression of electron-spin polarization decay at the QD ES due to the aforementioned selective relaxation of minority spins among the coupled subbands of the QDs is observed. With the increase in temperature, thermal activation of electron spins among QDs induces a faster decay of electron-spin polarization during light emission from the QD ESs. Above $140 \mathrm{~K}$, reinjection of depolarized electron spins from barriers after thermal escape from the QDs accelerates the electron-spin polarization decay. It was determined that the temperature dependences of spin dynamics depend on the areal QD density, i.e., coupling strength of the electron wavefunctions among the QDs.

\section{EXPERIMENTAL PROCEDURES}

Two QD samples were used in a previous study. ${ }^{8}$ These samples were grown via molecular beam epitaxy on GaAs(100) substrates. Two types of $\mathrm{In}_{0.5} \mathrm{Ga}_{0.5}$ As QDs with areal densities of $3.5 \times 10^{10}$ and $8.1 \times 10^{10} \mathrm{~cm}^{-2}$ were grown [see the inset in Fig. 1(a)]. Further details can be found in Ref. 8. Circularly polarized timeresolved photoluminescence (PL) was measured from $6 \mathrm{~K}$ to $200 \mathrm{~K}$ using a previously described method. ${ }^{8}$ A mode-locked Ti:Sapphire pulsed laser with a repetition rate of $80 \mathrm{MHz}$ and a pulse width of $<100 \mathrm{fs}$ was used as the excitation source. The diameter of the excitation laser spot was approximately $0.1 \mathrm{~mm}$. In order to make the number of injected carriers per QD identical, excitation powers for lower- and higher-density QDs were set to 6 and $10 \mathrm{~mW}$, respectively. Here, note that the excitation power for lower-density QDs was relatively higher taking into account that the injection efficiency of carriers into QDs becomes lower with the decrease in areal QD density. ${ }^{13}$ The excitation energy was tuned to $1.55 \mathrm{eV}$ to generate spin-polarized carriers in the GaAs barriers. The initial degree of electron-spin polarization generated in the GaAs barriers was $50 \%$ according to the optical selection rule. ${ }^{14}$ Although the streak camera used has a low detection sensitivity in the energy range of less than $1.4 \mathrm{eV}$, a calibration for the detection sensitivity was not performed. Circular polarization degree (CPD) values and their decay times, which are investigated in this study, are independent of the calibration.

\section{RESULTS AND DISCUSSION}

Figures 1(a) and 1(b) show the time-integrated circularly polarized PL spectra and the corresponding CPD for the lowerdensity QD (LQD) and higher-density QD (HQD) samples measured at $20 \mathrm{~K}$ and $180 \mathrm{~K}$. The CPD of PL is defined as $\mathrm{CPD}=\left(I_{\sigma+}-I_{\sigma-}\right) /\left(I_{\sigma+}+I_{\sigma-}\right)$ for circularly polarized PL intensities $I_{\sigma \pm}$, reflecting the electron-spin polarization at emissive states. The black solid lines show typical PL spectra of QD ground states (GSs) at low excitation power, which were measured using another detector with high sensitivity for the lower-energy side (down to $1.1 \mathrm{eV}$ ). The gray solid lines in Fig. 1(a) show typical PL spectra under strong excitation power. The PL components from the QD ESs above $1.30 \mathrm{eV}$ become more dominant with increasing excitation power above several milliwatts. The insets in Fig. 1(a) show atomic force microscopy (AFM) images of the LQD and HQD, in which the QD chains originated from the slightly misoriented GaAs substrates are observed for both samples. ${ }^{8,15}$ The average distance between the QD centers is estimated to be $60 \mathrm{~nm}$ and $30 \mathrm{~nm}$. When the center-to-center distance is smaller than $40 \mathrm{~nm}$, electron
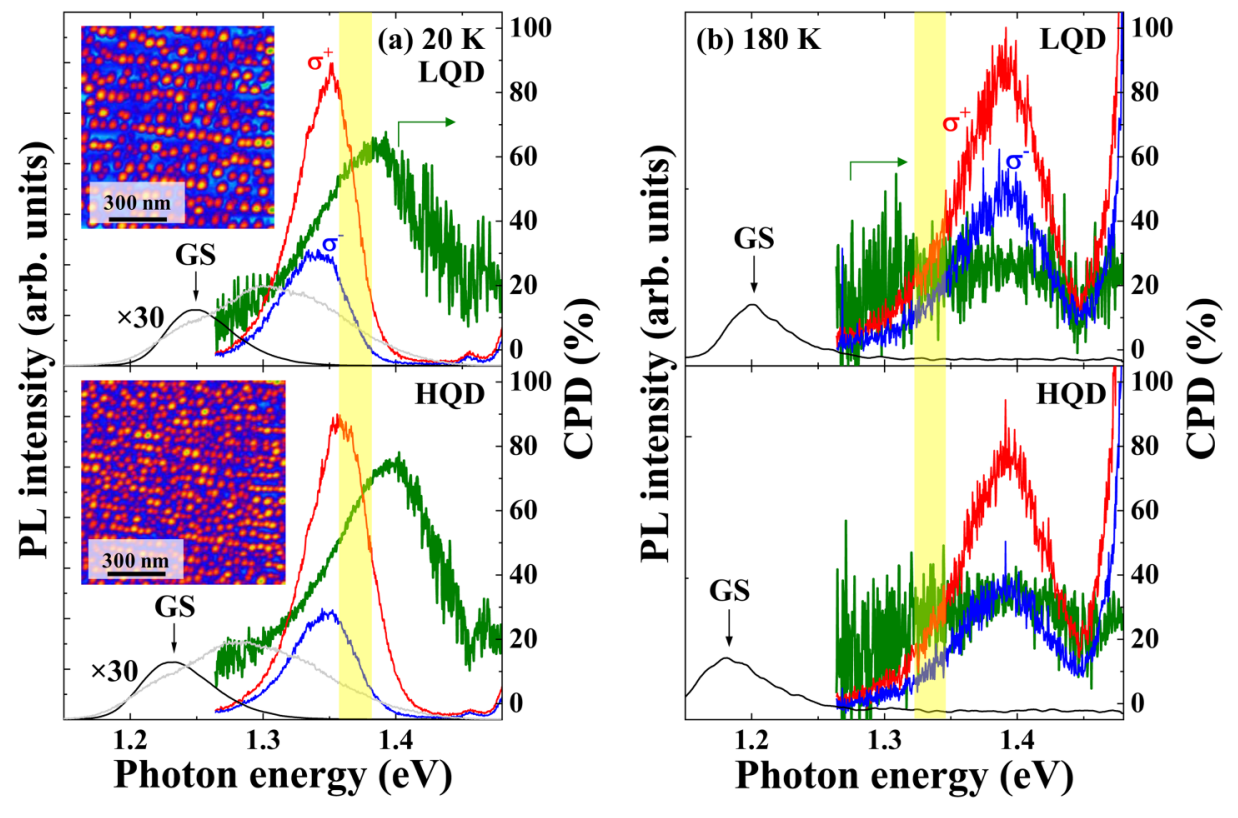

FIG. 1. Circularly polarized PL spectra and the corresponding CPD for LQD (upper) and HQD (lower) at (a) $20 \mathrm{~K}$ and (b) $180 \mathrm{~K}$. The insets in Fig. 1(a) show AFM images of the QDs. The PL spectra of the QD ground state (GS) are represented using black solid lines, for which the PL peak positions are identified using arrows. The gray solid lines in (a) show typical PL spectra under strong excitation power. The yellow rectangles show the $\mathrm{QD}$ excited states. 
wavefunction coupling among neighboring QDs becomes significant. The redshift of the GS peak energy for HQD compared to LQD may be attributed to the formation of extended states or minibands due to the strong coupling among QDs. ${ }^{16}$ Both QDs exhibit a lowerenergy shift of GS-PL with increasing temperature, which can be explained based on Varshni's law using the parameters of $\mathrm{In}_{0.5} \mathrm{Ga}_{0.5} \mathrm{As} .{ }^{17}$ For spin-dependent properties, HQD exhibits slightly higher CPD values compared to LQD for ESs above $1.36 \mathrm{eV}$. At the $\mathrm{ES}$ indicated by the yellow rectangles, the average $\mathrm{CPD}$ values are $58 \%$ and $60 \%$ for LQD and HQD, respectively. At $180 \mathrm{~K}$, the average CPD values decreased to $28 \%$ and $24 \%$. In this case, the analyzed energy ranges of the ES are shifted according to the aforementioned Varshni's law.

Figures 2(a)-2(c) show the circularly polarized PL time profiles and the corresponding CPD for QD ES represented by yellow rectangles in Fig. 1, measured at $20 \mathrm{~K}, 100 \mathrm{~K}$, and $180 \mathrm{~K}$. The analyzed energy ranges of the ES shift with a change in temperature according to Varshni's law. ${ }^{17}$ Single-exponential decay fitting of the time-dependent CPD was conducted (see the solid lines). Here, the data points of CPD in the latter half of the time region where the PL intensity is about $20 \%$ or less of the peak intensity are not included in the fitting range as the fluctuation of data points is large due to the low $\mathrm{S} / \mathrm{N}$ ratio, except the results of HQD measured at $6-90 \mathrm{~K}$ where two components for $\mathrm{CPD}$ decay were observed. The circularly polarized PL spectra and the corresponding CPD values for the QD ES are apparently identical for the two samples, whereas the CPD decay behavior was different. At $20 \mathrm{~K}$, a single component of the CPD decay $\left(\tau_{C P D}=0.48 \mathrm{~ns}\right)$ was observed for LQD and the resulting spin relaxation time of $0.96 \mathrm{~ns}$ is in good agreement with the previously reported value for conventional InGaAs QD ensembles. ${ }^{4,78}$ By contrast, the HQD exhibited two components for CPD decay. The second (shorter) CPD decay is identical to that of LQD, whereas a temporary long CPD decay $\left(\tau_{C P D}=0.68 \mathrm{~ns}\right)$ can be seen in the initial time region (black broken line). This temporary long CPD decay was clearly observed at $6-90 \mathrm{~K}$ for HQD. This temporary suppression of electron-spin polarization decay at the QD ES originates from the selective relaxation of minority spins from the emissive QD ESs to lower-energy states in neighboring QDs via wavefunction coupling. ${ }^{8-10}$ This result demonstrates the presence of interdot electron spin transfer for HQD. At $100 \mathrm{~K}$, the CPD decay time for LQD decreased from $0.48 \mathrm{~ns}$ to $0.35 \mathrm{~ns}$. By contrast, for HQD, the temporary long CPD decay almost disappeared and the CPD decay time decreased significantly to $0.27 \mathrm{~ns}$. At $180 \mathrm{~K}$, HQD exhibits faster CPD decay $\left(\tau_{C P D}=0.12 \mathrm{~ns}\right)$ compared to that of LQD $\left(\tau_{C P D}=0.15 \mathrm{~ns}\right)$.

Figure 3 shows the temperature dependence of $\tau_{C P D}$ for LQD and HQD. As previously described, the first component of $\tau_{C P D}$ for HQD at low temperature becomes longer because of spin-selective transfer among the QDs. Therefore, the second (shorter) component of $\tau_{C P D}$ is plotted as the net $\tau_{C P D}$. The red and blue solid lines represent the results of Arrhenius fitting for the temperaturedependent $\tau_{C P D}$ of LQD and HQD, respectively. In this case, single- and double-component fittings were performed for LQD and HQD. For LQD, $\tau_{C P D}$ is a constant in the temperature range of $6 \mathrm{~K}-70 \mathrm{~K}$ and decreases drastically above $70 \mathrm{~K}$, whereas $\tau_{C P D}$ for HQD gradually decreased from $6 \mathrm{~K}$ to $70 \mathrm{~K}$ and significantly decreased above $70 \mathrm{~K}$. In this case, we performed three-dimensional calculations for the conduction-band profiles and the eigenstates for two QDs that are separated by $30 \mathrm{~nm}$ between QD centers, for which the base lengths of the QDs are set to $20 \mathrm{~nm}$ and $16 \mathrm{~nm}$ based on our AFM analysis of the HQD. These calculations were conducted using the nextnano software package. ${ }^{19}$ According to
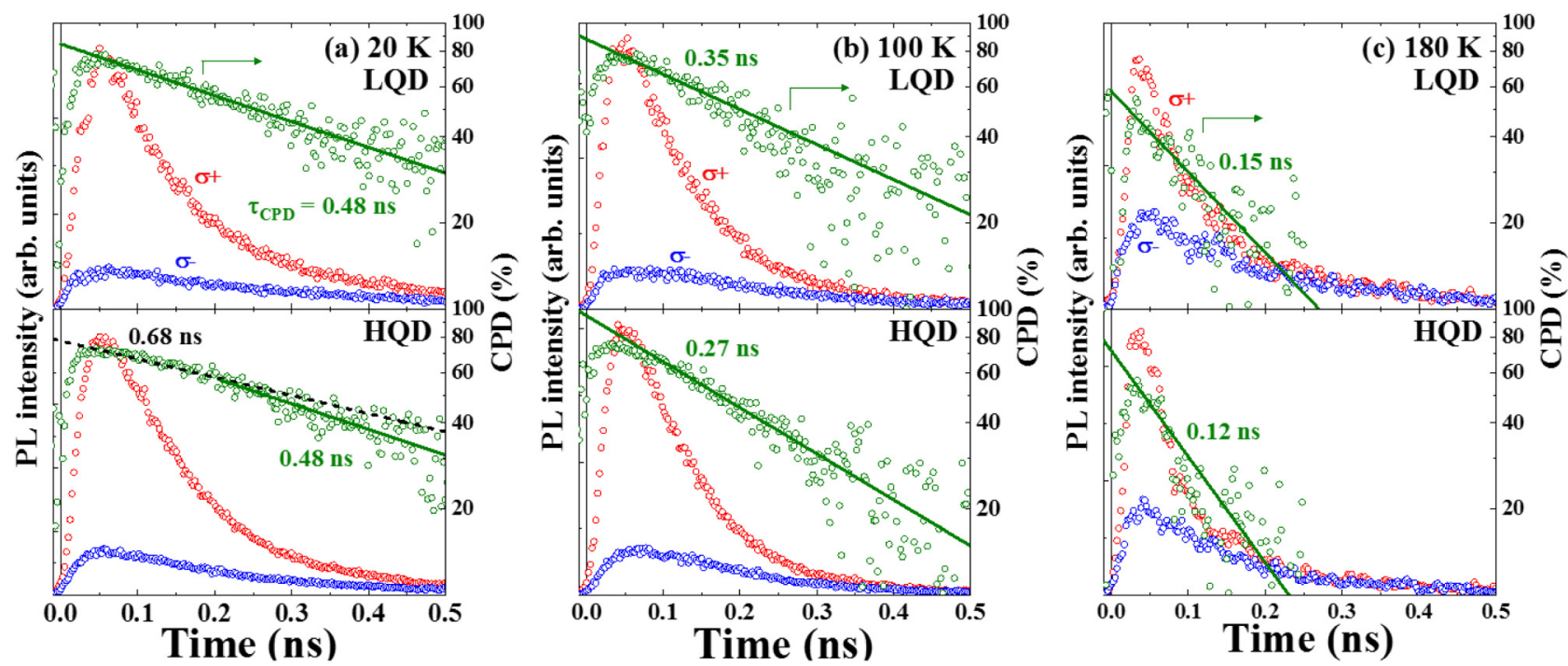

FIG. 2. Circularly polarized PL time profiles and the corresponding CPD for the QD ES (represented by yellow rectangles in Fig. 1) at (a) $20 \mathrm{~K}$, (b) $100 \mathrm{~K}$, and (c) $180 \mathrm{~K}$. The solid lines show the single-exponential decay fittings for the time-dependent CPD. The temporary long CPD decay is seen in the initial time region for HQD at $20 \mathrm{~K}$ (the black broken line). 


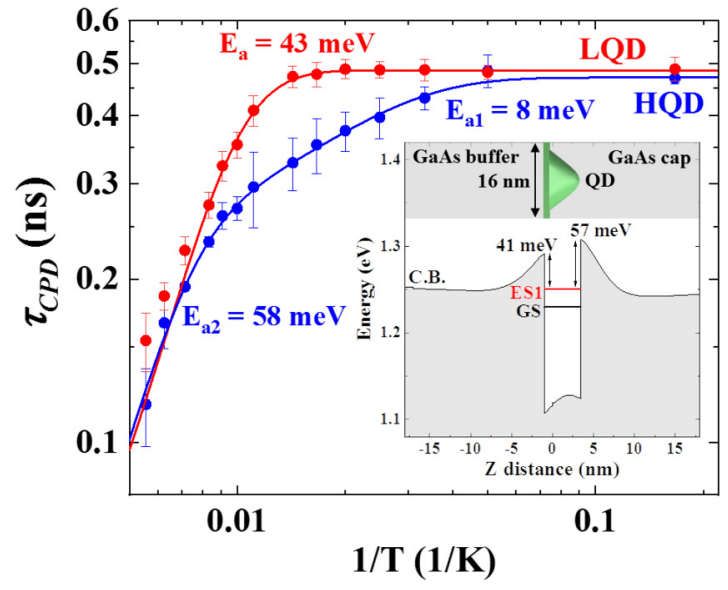

FIG. 3. $\tau_{C P D}$ of $L Q D$ (red circles) and HQD (blue circles) as a function of temperature. The red and blue solid lines indicate the Arrhenius fitting results. The inset shows the conduction-band profile and the eigenstates for a QD with a base length of $16 \mathrm{~nm}$ along the stacking direction, where the influence of strain on the band structure is considered.

the calculation result, the energy difference between the first ESs of the two QDs is $7 \mathrm{meV}$, which agrees well with the smaller thermal activation energy $E_{a 1}$ of $8 \mathrm{meV}$ for HQD. This result indicates that thermal activation of electron spins from lower-energy ESs to higher-energy ESs can occur between neighboring QDs. In this case, both majority and minority spins can be thermally excited to higher-energy QD states, although minority spins are selectively relaxed to lower-energy QD states at low temperatures. As a result, the thermal activation of minority spins can lead to a faster decay of electron-spin polarization at the QD ES. By contrast, the result for the Arrhenius fitting of LQD shows only the thermal activation energy of $43 \mathrm{meV}$. The small activation energy of several milli-electron volts in HQD was not observed for LQD. This result demonstrates that thermal activation of electron spin among QDs is much less dominant for LQD, due to the longer separation between QDs, resulting in a lower tunneling probability of electron spins between the QDs. At temperatures above $70 \mathrm{~K}$, the second component of the thermal activation energy $\mathrm{E}_{\mathrm{a} 2}=58 \mathrm{meV}$ appears for HQD. The inset shows typical results for three-dimensional calculations for the conduction-band profiles and the eigenstates of QDs with a base length of $16 \mathrm{~nm}$ along the stacking direction. In this calculation, the influence of strain on the local band structure is considered based on the theoretical report of strained InAs/GaAs QDs. ${ }^{20}$ If three-dimensional nanostructures are placed inside a crystalline matrix with different lattice constants, the resulting strain is not restricted to the inside of the nanostructure but continues into the surrounding structure. ${ }^{21}$ Consequently, the presence of strain increases the local band edge in the vicinity of QDs. The calculated energy height differences between the QD ES and barriers are $41 \mathrm{meV}$ and $57 \mathrm{meV}$. The difference between the two values is attributed to the strain generated above and below the QD. These values are close to the thermal activation energies of $43 \mathrm{meV}$ and $58 \mathrm{meV}$ obtained for LQD and HQD. This indicates that at high temperatures, the electron-spin polarization decay at QD ESs can be accelerated by reinjection of electrons with degraded spin polarizations after thermal escape from the QD ESs. This is discussed later.

For a more detailed understanding of the temperature dependence of spin dynamics in coupled QDs, a rate-equation fit analysis that considered the electron-spin transfer among QDs was conducted based on a previous report. ${ }^{22}$ Figure 4(a) shows a schematic model of the rate equation, considering the electron-spin transfer between a higher-energy state of QD1 and a lower-energy state of the adjacent QD2. In this case, the ES detected by the time-resolved PL is expressed as the QD1 state. In this model, $a$ is defined as a spin conservation factor during the spin injection process from a barrier into the QD. The parameters $\tau_{r}$ and $\tau_{s}$ denote the time constants for radiative recombination and electron-spin relaxation at the QD ES, respectively. The parameters of $\tau_{t r}^{\text {eff }}$ are effective time constants of the interdot spin transfer from QD1 to QD2, which includes thermal activation of electron spins from QD2 to QD1. In this work, electron-spin relaxation during spin transfer between QDs is not considered. In this case, the parameter $\tau_{r}$ is set to $0.3 \mathrm{~ns}$ regardless of temperature, based on previous reports. ${ }^{7,22}$ Figures 4 (b) and 4 (c) show the circularly polarized PL time profiles and the corresponding CPD for LQD and HQD measured at $20 \mathrm{~K}, 100 \mathrm{~K}$, and $180 \mathrm{~K}$. The best-fitted calculations of the rate equation are also shown. The characteristic time responses of $\mathrm{CPD}$, as well as the circularly polarized transient $\mathrm{PL}$ intensities, can be well-expressed. A combination of two fitting parameters, $\tau_{t r}^{e f f}$ and $\tau_{s}$, can explain the PL decay features with both polarization and the resulting CPD transients.

The temperature dependence of $\tau_{t r}^{e f f}$ for LQD and HQD deduced from the rate equation fitting is shown in Fig. 5. We can separate the spin-dynamics behavior at QD ES into three temperature regions: low temperatures below $70 \mathrm{~K}$, middle temperatures from $70 \mathrm{~K}$ to $140 \mathrm{~K}$, and high temperatures above $140 \mathrm{~K}$. At $6 \mathrm{~K}$, $\tau_{t r}^{e f f}$ of $0.05 \mathrm{~ns}$ for HQD is much faster than that of $0.32 \mathrm{~ns}$ for LQD. The faster $\tau_{t r}^{e f f}$ is due to the significant spin transfer among the QDs due to the significantly stronger coupling of electron wavefunctions. This spin transfer among the QDs induces temporary suppression of electron-spin polarization decay owing to the selective relaxation of minority spins among the QDs. With the increase in temperature from $6 \mathrm{~K}$ to $70 \mathrm{~K}$, LQD shows a gradual decrease in $\tau_{t r}^{e f f}$, whereas that of HQD is constant in this temperature range. In this case, the shrinkage of the GaAs bandgap with temperature is larger than that of $\operatorname{In}_{0.5} \mathrm{Ga}_{0.5}$ As. Therefore, the energy barrier height among laterally aligned QDs for electron spins becomes lower, leading to the coupling of electron wavefunctions among the QDs. As a result, $\tau_{t r}^{\text {eff }}$ decreases with the increase in temperature, even in LQD. This behavior is clearly observed for LQD. By contrast, no such behavior is observed for HQD. We anticipate that thermal activation $\left(E_{a 1}=8 \mathrm{meV}\right)$ of electron spins from lowerenergy states to higher-energy states among HQD-QDs can prevent a decrease in $\tau_{t r}^{\text {eff }}$ with temperature although the electron wavefunction coupling becomes stronger. For the middle temperature range of $70 \mathrm{~K}-140 \mathrm{~K}$, both LQD and HQD show a gradual increase in $\tau_{t r}^{\text {eff }}$ with increasing temperature. In this temperature range, thermal activation of electron spins from lower-energy states to higherenergy states inside a QD becomes more dominant with the increase in temperature. Consequently, $\tau_{t r}^{e f f}$, corresponding to the spin transfer time from higher-energy states to lower-energy states 
(a)
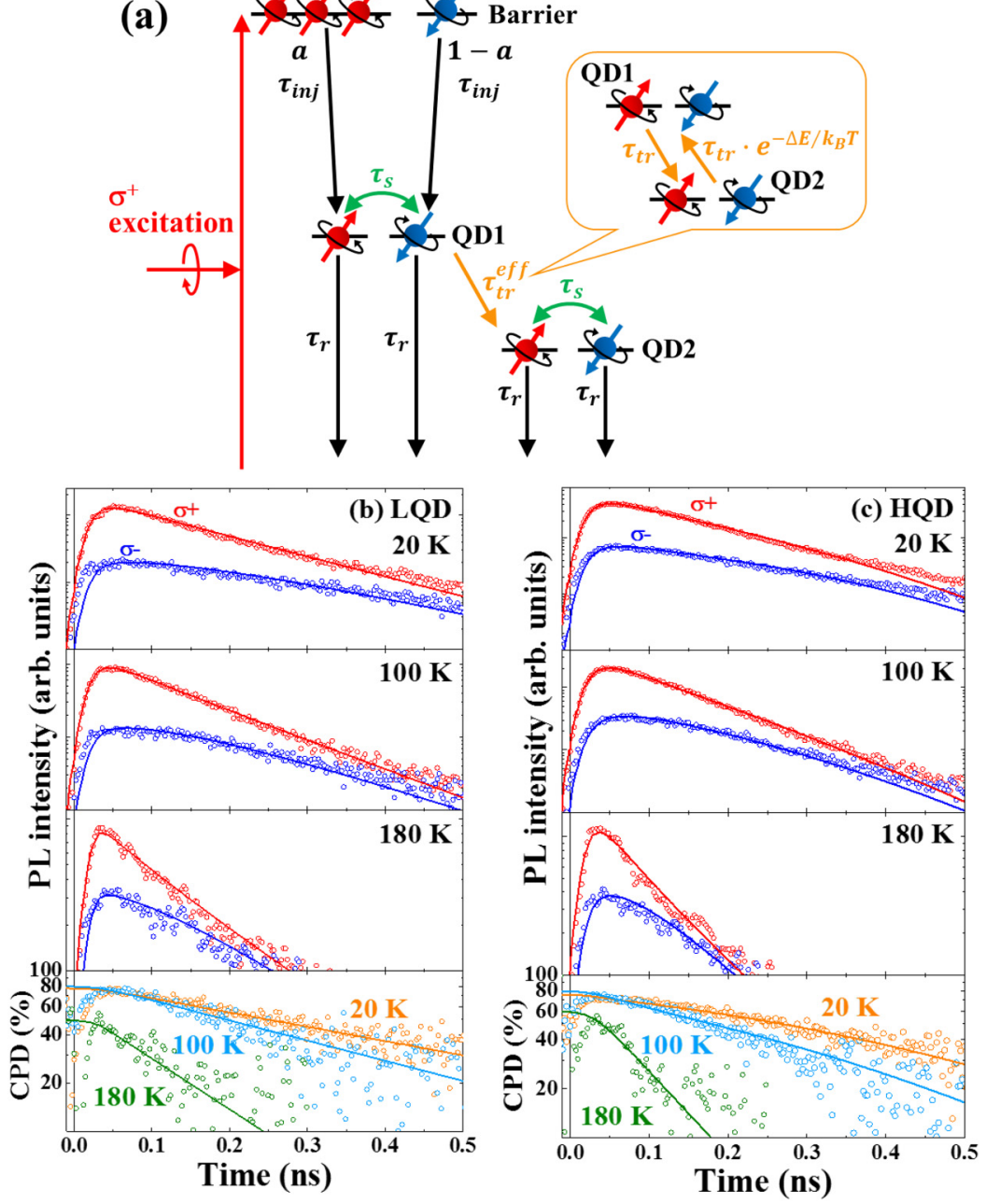

FIG. 4. (a) Schematic model of rate equations including interdot spin transfer between higher-energy state QD1 and the neighboring lower-energy state QD2. Circularly polarized PL time profiles and the corresponding CPD for (b) LQD and (c) HQD measured at $20 \mathrm{~K}, 100 \mathrm{~K}$, and $180 \mathrm{~K}$. The solid lines show the best-fitted calculations of the rate equations.

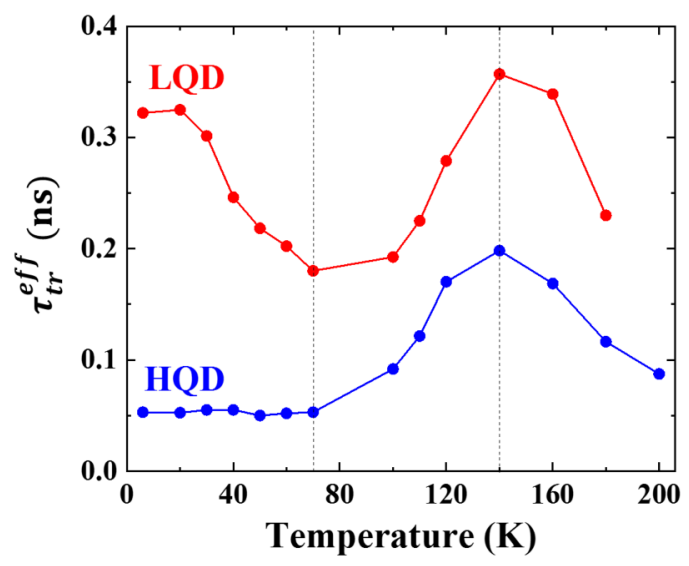

FIG. 5. $\tau_{t r}^{\text {eff }}$ of $L Q D$ (red circles) and HQD (blue circles) as a function of temperature, deduced from the rate equation fitting. among QDs, becomes longer. A faster $\tau_{t r}^{e f f}$ for HQD is observed because the interdot relaxation of electron spins remains more dominant. At high temperatures above $140 \mathrm{~K}$, a decrease in $\tau_{t r}^{\text {eff }}$ with temperature was commonly observed for LQD and HQD. At a high temperature, electron spins can thermally escape from the QD ESs to a three-dimensional barrier. In the case of HQD, electron spins can be thermally activated stepwise from one QD state to the next higher state. ${ }^{23}$ Therefore, thermal escape of electron spins from QD to the barriers becomes more dominant for HQD compared to LQD.

Figure 6 shows the temperature dependence of $\tau_{s}$ for LQD and HQD deduced from the rate-equation fitting. The large difference in $\tau_{s}$ between LQD and HQD at low temperatures below $70 \mathrm{~K}$ is observed. The faster $\tau_{s}$ for HQD can be attributed to the increased dimensionality of the quantum structure due to the stronger coupling of electron wavefunctions among laterally coupled QDs, in which a more significant electron-spin relaxation mechanism is in effect. At low temperatures, the Bir-Aronov-Pikus spin relaxation is known to act. $^{24}$ Electron migration implies that there is stronger coupling 


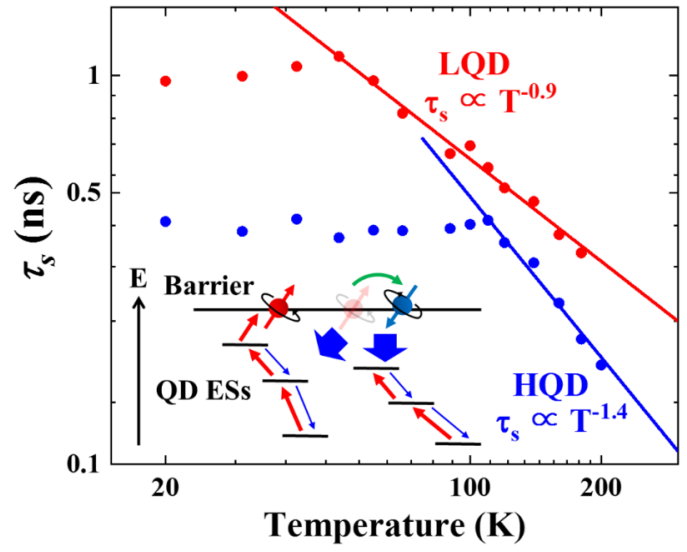

FIG. 6. $\tau_{s}$ of LQD (red circles) and HQD (blue circles) as a function of temperature. The red and blue solid lines show the $T^{-0.9}$ and $T^{-1.4}$ dependences, respectively. The inset shows a schematic model of electron-spin dynamics at high temperatures. The red and thin blue arrows represent the thermal excitation and relaxation of electron spins among the QDs, respectively. The green and thick blue arrows represent the electron-spin relaxation in the barrier and the reinjection of electron spins from the barrier into the QD ESs, respectively.

with holes. Therefore, the second component of $\tau_{C P D}$ discussed in Fig. 2(a) should include both the "net" electron-spin relaxation in HQD and the effect of interdot relaxation of minority spins, because the net electron-spin relaxation time of HQD is faster than that of LQD. With the increase in temperature, for LQD, $\tau_{s}$ starts to decrease above $50 \mathrm{~K}$ and exhibits a $T^{-0.9}$ dependence. This result is relatively close to the $\mathrm{D}^{\prime}$ yakonov-Perel ${ }^{\prime}$ (DP) electron-spin relaxation in a quantum well, ${ }^{25}$ which exhibits a $T^{-1}$-dependence. By contrast, HQD shows a $T^{-1.4}$ dependence. The latter result is relatively closer to the DP electron-spin relaxation in bulk, ${ }^{25}$ which exhibits a $T^{-3}$ dependence. In the case of HQD, thermal excitation of electron spins from QD ES to barriers occurs more dominantly compared to LQD because electron spins can thermally escape from QDs via stepwise ladderlike activation among smaller QDs (see the inset in Fig. 6). In this case, electron spins should be thermally excited to barriers via the ESs of small QDs, which are energetically close to the barriers. In conclusion, the following behavior can occur for HQD at high temperatures, as shown in the inset in Fig. 6. Firstly, electron spins thermally escape from QD ESs to barriers, and subsequently, electron-spin relaxation proceeds with accelerated spin-relaxation times in the three-dimensional barrier. Next, these electron spins are rapidly reinjected from the barrier to the QD ESs. As a result, the electron-spin polarization decay from QD-ES emission can be accelerated at high temperatures. These results indicate that the temperature dependence of $\tau_{s}$ at the QD ES for HQD more prominently reflects the electron-spin relaxation in a three-dimensional barrier. This finding clearly indicates that the suppression of the reinjection of electron spins from barriers in the high-density QD system is crucial for maintaining high electron-spin polarization during light emission from QD ES at high temperatures. However, deeper QD potentials induce multiple phonon emissions during relaxation from the barrier to the QD-ESs. The participation of many phonons in spin-polarized electron relaxation from the barrier can weaken the spin polarization. ${ }^{26}$ Therefore, an appropriate design of the quantum structure surrounding each QD in the high-density QD system is highly desired, such as the barrier height and the interdot tunneling rate. An in-depth understanding of spin relaxation induced by thermal excitation during interdot transfer into a barrier is necessary to achieve room-temperature operation of spin-functional QD optical devices.

\section{SUMMARY}

We studied the temperature dependence of spin dynamics in the ESs of laterally coupled high-density $\operatorname{In}_{0.5} \mathrm{Ga}_{0.5}$ As QDs. At low temperatures below $100 \mathrm{~K}$, a temporary suppression of electronspin polarization decay due to spin-selective interdot transfer was observed, which was the same as that reported in a previous work at $6 \mathrm{~K}$. In the temperature range of $20 \mathrm{~K}-140 \mathrm{~K}$, thermal activation of minority spins from lower-energy states to higher-energy states among QDs leads to a faster decay of electron-spin polarization for the QD ESs. At high temperatures above $140 \mathrm{~K}$, strong coupling of electron wavefunctions among QDs induces further decay of electronspin polarization due to the reinjection of depolarized electron spins from barriers, after ladderlike thermal escape from the QD ESs. An appropriate design of the quantum structure surrounding each QD, such as the barrier height and the interdot tunneling rate in a highdensity QD system, is necessary for room-temperature operation of spin-functional QD optical devices. Moreover, this is also necessary to better understand the spin relaxation induced by thermal excitation during interdot transfer into a barrier.

\section{ACKNOWLEDGMENTS}

This work was supported by the Japan Society for the Promotion of Science (JSPS) under Grant Nos. 16H06359, 19K15380, and 19H05507.

\section{REFERENCES}

${ }^{1}$ A. V. Khaetskii and Y. V. Nazarov, "Spin relaxation in semiconductor quantum dots," Phys. Rev. B 61, 12639 (2000).

${ }^{2}$ P. Borri, W. Langbein, S. Schneider, U. Woggon, R. L. Sellin, D. Ouyang, and D. Bimberg, "Ultralong dephasing time in InGaAs quantum dots," Phys. Rev. Lett. 87, 157401 (2001).

${ }^{3}$ M. Paillard, X. Marie, P. Renucci, T. Amand, A. Jbeli, and J. M. Gérard, "Spin relaxation quenching in semiconductor quantum dots," Phys. Rev. Lett. 86, 1634 (2001).

${ }^{4}$ A. Tackeuchi, R. Ohtsubo, K. Yamaguchi, M. Murayama, T. Kitamura, T. Kuroda, and T. Takagahara, "Spin relaxation dynamics in highly uniform InAs quantum dots,” Appl. Phys. Lett. 84, 3576 (2004).

${ }^{\mathbf{5}}$ T. Amano, T. Sugaya, and K. Komori, "Characteristics of $1.3 \mu \mathrm{m}$ quantum-dot lasers with high-density and high-uniformity quantum dots," Appl. Phys. Lett. 89, 171122 (2006).

${ }^{\boldsymbol{\sigma}_{\mathrm{C}}}$ K. Watanabe, T. Akiyama, Y. Yokoyama, K. Takemasa, K. Nishi, Y. Tanaka, M. Sugawara, and Y. Arakawa, "Growth of high-densityl.06- $\mu \mathrm{m}$ InGaAs/GaAs quantum dots for high gain lasers by molecular beam epitaxy,” J. Cryst. Growth 378, 627 (2013).

${ }^{7}$ T. Yamamura, T. Kiba, X. J. Yang, J. Takayama, A. Subagyo, K. Sueoka, and A. Murayama, "Growth-temperature dependence of optical spin-injection dynamics in self-assembled InGaAs quantum dots,” J. Appl. Phys. 116, 094309 (2014). 
${ }^{8}$ S. Hiura, K. Takeishi, M. Urabe, K. Itabashi, J. Takayama, T. Kiba, K. Sueoka, and A. Murayama, "Interdot spin transfer dynamics in laterally coupled excited spin ensemble of high-density InGaAs quantum dots," Appl. Phys. Lett. 113, 023104 (2018).

${ }^{9}$ S. Hiura, M. Urabe, K. Takeishi, K. Itabashi, J. Takayama, T. Kiba, K. Sueoka, and A. Murayama, "Interdot carrier and spin dynamics in a two-dimensional high-density quantum-dot array of InGaAs with quantum dots embedded as local potential minima," Semicond. Sci. Technol. 34, 025001 (2019).

${ }^{10}$ S. Hiura, S. Saito, J. Takayama, T. Kiba, and A. Murayama, "Layer-selective spin amplification in size-modulated quantum nanocolumn," Appl. Phys. Lett. 115, 013102 (2019).

${ }^{11}$ A. Tackeuchi, Y. Nakata, S. Muto, Y. Sugiyama, T. Usuki, Y. Nishikawa, N. Yokoyama, and O. Wada, "Time-Resolved study of carrier transfer among InAs/GaAs multi-coupled quantum dots," Jpn. J. Appl. Phys. 34, L1439 (1995).

${ }^{12}$ G. G. Tarasov, Y. I. Mazur, Z. Y. Zhuchenko, A. Maaßdorf, D. Nickel, J. W. Tomm, H. Kissel, C. Walther, and W. T. Masselink, "Carrier transfer in selfassembled coupled InAs/GaAs quantum dots," J. Appl. Phys. 88, 7162 (2000).

${ }^{13}$ F. V. de Sales, J. M. R. Cruz, S. W. da Silva, M. A. G. Soler, P. C. Morais, M. J. da Silva, A. A. Quivy, and J. R. Leite, "Carrier kinetics in quantum dots through continuous wave photoluminescence modeling: A systematic study on a sample with surface dot density gradient," J. Appl. Phys. 94, 1787 (2003).

${ }^{14} \mathrm{~F}$. Meier and B. Zakharchenya, Optical Orientation (Elsevier, North Holland, Amsterdam, 1984).

${ }^{15} \mathrm{M}$. Kitamura, M. Nishioka, J. Oshinowo, and Y. Arakawa, "In situ fabrication of self-aligned InGaAs quantum dots on GaAs multiatomic steps by metalorganic chemical vapor deposition," Appl. Phys. Lett. 66, 3663 (1995).

${ }^{16}$ S. Lan, K. Akahane, K.-Y. Jang, T. Kawamura, Y. Okada, M. Kawabe, T. Nishimura, and O. Wada, "Two-dimensional $\mathrm{In}_{0.4} \mathrm{Ga}_{0.6} \mathrm{As} / \mathrm{GaAs}$ quantum dot superlattices realized by self-organized epitaxial growth," Jpn. J. Appl. Phys. 38, 2934 (1999).
${ }^{17}$ S. Paul, J. B. Roy, and P. K. Basu, "Empirical expressions for the alloy composition and temperature dependence of the band gap and intrinsic carrier density in $\mathrm{Ga}_{\mathrm{x}} \mathrm{In}_{1-\mathrm{x}} \mathrm{As}$," J. Appl. Phys. 69, 827 (1991).

${ }^{18}$ T. Kiba, X. J. Yang, T. Yamamura, Y. Kuno, A. Subagyo, K. Sueoka, and A. Murayama, "Temperature dependence of the dynamics of optical spin injection in self-assembled InGaAs quantum dots," Appl. Phys. Lett. 103, 082405 (2013).

${ }^{19}$ S. Birner, T. Zibold, T. Andlauer, T. Kubis, M. Sabathil, A. Trellakis, and P. Vogl, "Nextnano: General purpose 3-D simulations," IEEE Trans. Electron Devices 54, 2137 (2007).

${ }^{20} \mathrm{C}$. Pryor, "Eight-band calculations of strained InAs/GaAs quantum dots compared with one-, four-, and six-band approximations," Phys. Rev. B 57, 7190 (1998).

${ }^{21}$ A. Schliwa, G. Hönig, and D. Bimberg, Electronic Properties of III-V Quantum Dots (Springer International Publishing, Cham, 2014), pp. 57-85.

${ }^{22}$ K. Takeishi, S. Hiura, J. Takayama, K. Itabashi, M. Urabe, A. Washida, T. Kiba, and A. Murayama, "Persistent high polarization of excited spin ensembles during light emission in semiconductor quantum-dot-well hybrid nanosystems," Phys. Rev. Appl. 10, 034015 (2018).

${ }^{23} \mathrm{Z}$. Ma, K. Pierz, and P. Hinze, "Abnormal temperature behavior of photoluminescence from self-assembled InAs/AlAs quantum dots," Appl. Phys. Lett. 79, 2564 (2001).

${ }^{24}$ G. L. Bir, A. G. Aronov, and G. E. Pikus, "Spin relaxation of electrons due to scattering by holes," Sov. Phys. JETP 42, 705 (1976).

${ }^{25}$ R. S. Britton, T. Grevatt, A. Malinowski, R. T. Harley, P. Perozzo, A. R. Cameron, and A. Miller, "Room temperature spin relaxation in GaAs/ AlGaAs multiple quantum wells," Appl. Phys. Lett. 73, 2140 (1998).

${ }^{26}$ Y. Q. Huang, I. A. Buyanova, X. J. Yang, A. Murayama, and W. M. Chen, "Effect of a phonon bottleneck on exciton and spin generation in self-assembled $\mathrm{In}_{1-\mathrm{x}} \mathrm{Ga}_{\mathrm{x}} \mathrm{As}$ quantum dots," Phys. Rev. Appl. 9, 044037 (2018). 